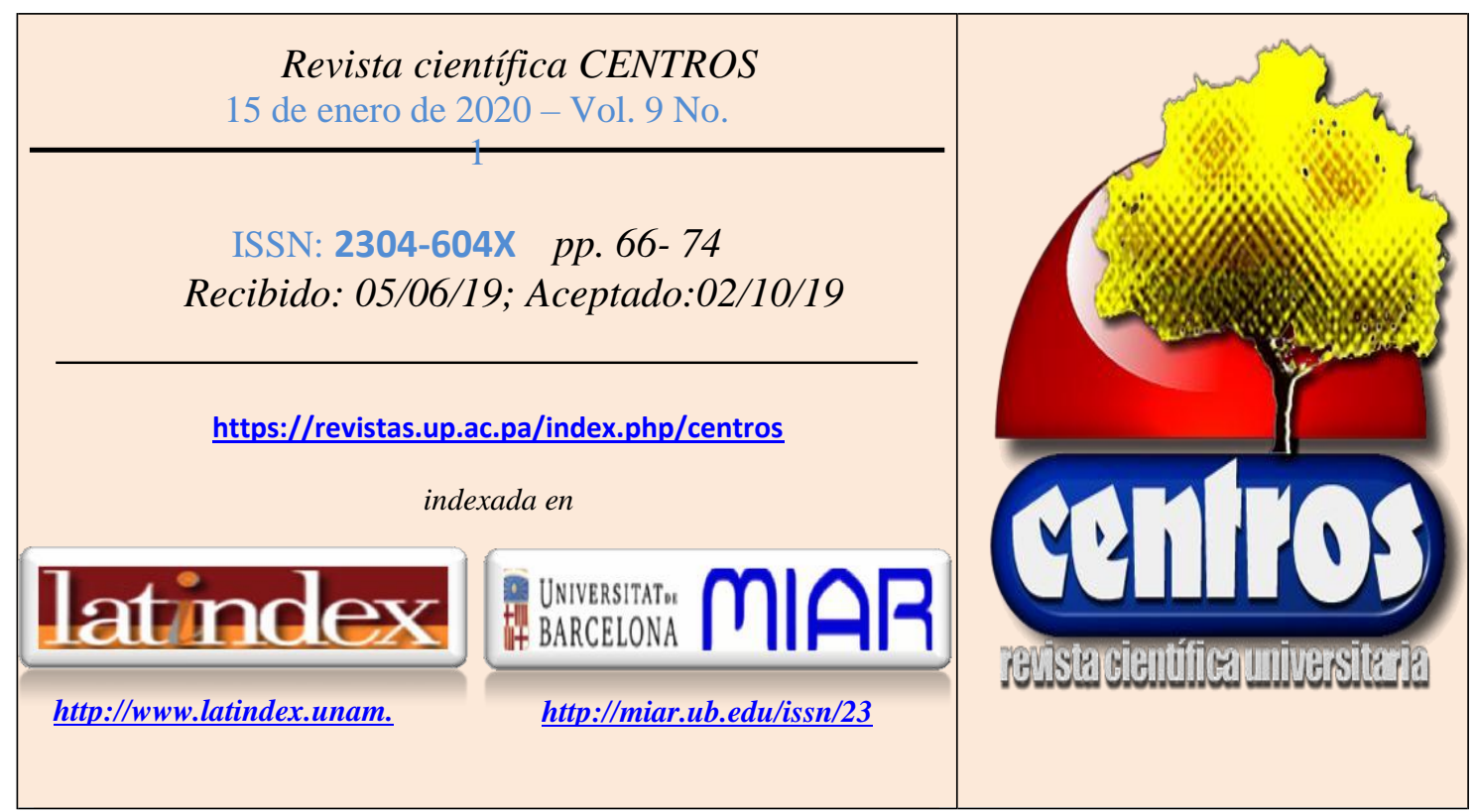

\title{
VINCULACIÓN DE UNIANDES - QUEVEDO Y SU IMPACTO EN EL DESARROLLO DE EMPRENDIMIENTOS EN LA PROVINCIA DE LOS RÍOS
}

\author{
LINKING UNIANDES - QUEVEDO AND ITS IMPACT ON THE \\ DEVELOPMENT OF ENTERPRISES IN THE PROVINCE OF LOS RÍOS \\ Lisenia Karina Baque Villanueva
}

Universidad Regional Autónoma de Los Andes.*uq.liseniabaque@uniandes.edu.ec

\section{RESUMEN}

En la actualidad las instituciones de educación superior tienen un gran compromiso social con la comunidad, ya que asiste en la solución de problemas socioeconómicos; es así que la universidad se establece como un ente que proporciona herramientas y formación técnica - administrativa de calidad para la generación de emprendimientos de negocios, unidades productivas y microempresas, articulándose a los grandes objetivos estratégicos de la matriz productiva del país contemplados en el Plan de Desarrollo Nacional Toda Una Vida. La presente investigación tuvo por objetivo analizar la vinculación de la Universidad Regional Autónoma de los Andes - Quevedo y el impacto que ésta ha tenido en la generación de emprendimientos en la Provincia de Los Ríos. Para realizar este estudio se aplicó el método cuali-cuantitativo e investigación bibliográfica, la población sujeta de análisis fueron 24 participantes del proyecto Impulso Joven promocionado por la Secretaría Técnica de Juventudes. El estudio permitió determinar que UNIANDES-Quevedo contribuyó en la elaboración del 69\% de planes de negocios que serán ejecutados en los cantones Quevedo, Valencia, Buena Fe y Mocache, favoreciendo a la productividad y competitividad de los actores y organizaciones de economía popular y solidaria. La IES en mención prioriza y atiende las necesidades de la población, proporcionando herramientas técnicas y administrativas para la generación de emprendimientos. Por esta razón los encuestados han mostrado total satisfacción en la participación de proyectos de vinculación ofertados, lo cual genera un impacto positivo para el desarrollo económico y social de los emprendedores de la provincia de Los Ríos.

Palabras claves: Compromiso Social, Desarrollo Empresarial, Emprendimiento, Vinculación. 


\begin{abstract}
At present, higher education institutions have a great social commitment to the community, as it assists in the solution of socioeconomic problems; Thus, the university is established as an entity that provides tools and technical-administrative quality training for the generation of business ventures, productive units and micro-enterprises, articulating the major strategic objectives of the productive matrix of the country contemplated in the Plan of National Development A Whole Life The objective of this research was to analyze the link between the Autonomous Regional University of the Andes - Quevedo and the impact it has had on the generation of enterprises in the Province of Los Ríos. To carry out this study, the qualitative-quantitative method and bibliographic research was applied; the population subject to analysis was 24 participants of the Young Impulse project promoted by the Youth Technical Secretariat.

The study allowed to determine that UNIANDES-Quevedo contributed in the elaboration of $69 \%$ of business plans that will be executed in the cantons Quevedo, Valencia, Buena Fe and Mocache, favoring the productivity and competitiveness of the actors and organizations of popular and solidary economy. The mentioned HEI prioritizes and attends to the needs of the population, providing technical and administrative tools for the generation of enterprises. For this reason, the respondents have shown total satisfaction in the participation of bonding projects offered, which generates a positive impact for the economic and social development of entrepreneurs in the province of Los Ríos.
\end{abstract}

Key words: Social Commitment, Business Development, Entrepreneurship, Linking. 


\section{INTRODUCCIÓN}

Las necesidades económicas por las que atraviesa América Latina y en especial nuestro país, hace que cada vez más la sociedad busque diversas formas de superación de la pobreza y bienestar de sus familias, a través de la generación de emprendimientos que contribuyan con el desarrollo productivo.

En los últimos años, la erradicación de la pobreza ha sido altamente prioritario para el Gobierno Nacional, por ello se están efectuando esfuerzos imperiosos, para incentivar a hombres y mujeres en el emprendimiento de microempresas.

Bajo este enfoque, para fomentar el crecimiento personal y colectivo de la comunidad en aspectos, económicos y sociales, es necesario motivar y guiar a las organizaciones de economía popular y solidaria para que logren plasmar sus ideas de negocios, generando oportunidades de empleo que ofrezcan confianza y un apropiado nivel de vida, contribuyendo así con el Plan Nacional de Desarrollo 2017-2021.

La universidad Regional Autónoma de los Andes Quevedo, durante su trayectoria ha dado muestra del compromiso social que se ha concretado en el servicio a la sociedad a través de la educación de tercer y cuarto nivel y que en la actualidad fortalece esta visión con proyectos concretos que benefician a la sociedad.

A través de la ejecución de proyectos de vinculación se pretende formar emprendedores, transformando la realidad a través de la motivación y formación necesaria para llevar a cabo sus proyectos mediante el diseño de un plan de negocios sostenible.

Por lo antes expuesto, esta investigación pretende analizar el impacto que ha tenido la generación de emprendimientos en la Provincia de Los Ríos, considerando que ésta es una capacidad de los seres humanos para salir adelante de manera novedosa y con ideas renovadas. (Jaramillo , 2008)

\section{MARCO TEÓRICO}

Para Maass \& Sabulsky (2015) "La enseñanza en las aulas universitarias ha ido modificando sus rígidas estructuras e incorporando paulatinamente nuevas maneras de pensar la práctica educativa". (pp.89).

La vinculación es la función que cumple la universidad con el medio externo. Se apoya en la docencia y la investigación para el diseño y desarrollo de proyectos que articulen los conocimientos científicos y técnicos con los saberes y experiencias del entorno. (De Aparicio , Chininin , \& Toledo , 2017)

La Universidad ecuatoriana busca servir a la Sociedad mediante proyectos sustentables e innovadores que permitan mejorar la calidad de vida de los habitantes, teniendo como instrumentos claves en este proceso a estudiantes y docentes, contribuyendo así al Plan Nacional Toda Una Vida 2017 - 2021.

La vinculación universitaria acoge diversos elementos que permiten relacionarla con el medio: 1) la idea de una perspectiva dialógica entre universidad y sociedad. 2) la perspectiva del aprendizaje desde el pensamiento complejo. 3) la necesidad de promover prácticas de enseñanza innovadoras que incluyan diversos escenarios donde es posible que el aprendizaje se produzca, en particular, las situaciones reales como espacios de enseñanza y aprendizaje. (Maass \& Sabulsky, 2015)

Cuando la universidad participa como agente de la cooperación para el desarrollo debe existir una estrategia institucional que considera esta materia como "un espacio para expresar su responsabilidad social, proyectar $\mathrm{y}$ transferir las capacidades y 
conocimientos de la universidad y obtener diferentes tipos de retornos", ejecutando proyectos de cooperación para el desarrollo acorde con las capacidades institucionales que se posean. (Gaete, 2011)

Unceta (como se citó en Gaete, 2011) afirma que la universidad en el contexto de la cooperación al desarrollo posee algunas responsabilidades de importancia, tales como otorgar una mayor importancia a la investigación y el debate sobre las condiciones del desarrollo a nivel mundial.

Estas responsabilidades de las universidades en materia de cooperación al desarrollo se conectan adecuadamente con los planteamientos que vinculan a la responsabilidad social universitaria (RSU) con la contribución que estas instituciones deben realizar tanto al necesario debate para alcanzar una mejor Sociedad. (Gaete, 2011)

Las instituciones de Educación Superior, tienen el deber de colaborar conjuntamente con instituciones del medio externo, en la atención prioritaria de aquellos en situación de pobreza y exclusión, mediante la ejecución de proyectos que permitan formar emprendedores con conocimientos teóricos y prácticos, capaces de dirigir sus propias pequeñas y medianas empresas, las cuales son indispensables para el desarrollo empresarial y económico de nuestro país.

Según Delfín \& Acosta (2016) manifiestan que "el desarrollo empresarial articula diferentes elementos con los que el empresario puede llevar a una organización hacia el logro de sus objetivos. Elementos como crecimiento económico, cultura empresarial, liderazgo, gestión del conocimiento e innovación".

Con referencia a lo anterior se puede mencionar que, el desarrollo empresarial permitirá a pequeños y grandes comerciantes del cantón Quevedo poder integrar la innovación y el recurso humano para la consecución de los objetivos organizacionales, así como también aprovechar las oportunidades del entorno para contribuir al crecimiento económico local y nacional.

Por lo antes expuesto, es necesario hacer referencia al emprendimiento, que en palabras de Calzada (2012) es una actividad que siempre ha tenido buena prensa y las personas que se deciden a emprender han sido calificadas como de primera clase por parte del resto de los ciudadanos que no se han atrevido a arriesgarse. Porque el emprendimiento siempre comporta riesgos sea cualesquiera la actividad en la que se emprenda.

La actividad emprendedora es un elemento imprescindible para entender el desarrollo económico de cualquier país. Ayuda a la previsión y ajuste de políticas públicas allí donde se necesita el impulso de nuevos proyectos y la generación de empleo. Emprender es poner en marcha un nuevo proyecto empresarial asumiendo el riesgo que éste conlleva, a cambio de obtener un beneficio empresarial. Es un motor de innovación, competitividad y crecimiento. (Blanco , Mercado , \& Prado , 2012)

En la actualidad no es fácil emprender, hay muchas causas para que esto ocurra, entre ellas la falta de motivación y decisión para la creación de un negocio, inadecuada utilización de herramientas administrativas, técnicas para la gestión de emprendimientos, desconocimiento de las ventajas de la asociatividad y el escaso recurso económico, mismas que tienen efectos importantes como las ideas de negocios no concretadas por falta de confianza y de conocimientos, desconocimiento del entorno competitivo y económico de los 
emprendimientos,

inadecuado

aprovechamiento de las ventajas de la asociatividad para el desarrollo de emprendimientos y la no ejecución de una actividad comercial.

En este sentido, con la finalidad de apoyar las buenas iniciativas que coadyuven al mejoramiento de las condiciones socioeconómicas de los actores de la economía popular y solidaria en el cantón Quevedo, existen programas y proyectos públicos que conjuntamente con la Universidad, han impulsado la ejecución de emprendimientos.

\section{MATERIALES Y MÉTODOS}

Para el desarrollo de la investigación, se aplicó el método cuali-cuantitativo, que permitirá describir los eventos y comportamientos de las personas estudiadas, así como también analizar los datos numéricos de la investigación. El universo de la investigación está compuesto por 24 emprendedores que habitan en los cantones de Quevedo, Valencia, Mocache, Quinsaloma, Ventanas y Buena Fe, mismos que integran el Proyecto Impulso Joven promocionado por la Secretaría Técnica de Juventudes, a los cuales se les aplicó la encuesta como instrumento de recolección de datos, que permitirá medir el impacto que tiene UNIANDES-QUEVEDO en la generación de emprendimientos. La investigación bibliográfica permitió analizar las decisiones de algunos autores relacionados a las variables de estudio.

\section{RESULTADOS}

Los resultados de la investigación exponen la impresión que tiene la UNIANDES- QUEVEDO en el desarrollo de emprendimientos en la Provincia de Los Ríos. Los beneficiarios de este proyecto corresponden al rango entre 18 a 29 años de edad.

Tabla 1

\section{Motivación para iniciar un emprendimiento}

\begin{tabular}{lcc}
\hline Descripción & Frecuencia & Porcentaje \\
\hline $\begin{array}{l}\text { Mejorar los } \\
\text { ingresos }\end{array}$ & 17 & $71 \%$ \\
$\begin{array}{l}\text { Independencia } \\
\text { económica }\end{array}$ & 5 & $21 \%$ \\
$\begin{array}{l}\text { Desarrollo del } \\
\text { sector }\end{array}$ & 2 & $8 \%$ \\
Total & $\mathbf{2 4}$ & $\mathbf{1 0 0 \%}$ \\
\hline
\end{tabular}

La tabla 1 expone que el $71 \%$ de los participantes encuestados manifiestan que su principal motivo para emprender es mejorar los ingresos económicos en sus hogares, el $21 \%$ indica que su deseo es tener independencia económica, mientras que el $8 \%$ emprenden para mejorar el desarrollo del sector en el cual habitan.

Tabla 2

Valoración de los proyectos de vinculación

\begin{tabular}{lcc}
\hline Descripción & Frecuencia & Porcentaje \\
\hline $\begin{array}{l}\text { Muy } \\
\text { satisfactorio }\end{array}$ & 22 & $92 \%$ \\
\hline $\begin{array}{l}\text { Satisfactorio } \\
\text { Poco }\end{array}$ & 2 & $8 \%$ \\
satisfactorio & 0 & $0 \%$ \\
Nada & 0 & $0 \%$ \\
Satisfactorio & & $\mathbf{1 0 0 \%}$ \\
\hline Total & $\mathbf{2 4}$ & \\
\hline
\end{tabular}

En la tabla 2 se muestra las personas encuestadas que valoran la participación de UNIANDES - QUEVEDO con respecto a los proyectos de vinculación con la sociedad, en la que el $92 \%$ indica que es muy satisfactorio, mientras que el $8 \%$ dice que es satisfactorio. Este resultado presenta claramente que la mencionada Institución de Educación 
Superior ha tenido total aceptación por parte de la comunidad en la provincia de Los Ríos.

Tabla 3

Calidad de las actividades de Vinculación

\begin{tabular}{lcc}
\hline Descripción & Frecuencia & Porcentaje \\
\hline $\begin{array}{l}\text { Muy } \\
\text { satisfactorio }\end{array}$ & 20 & $83 \%$ \\
Satisfactorio & 4 & $17 \%$ \\
$\begin{array}{l}\text { Poco } \\
\text { satisfactorio }\end{array}$ & 0 & $0 \%$ \\
$\begin{array}{l}\text { Nada } \\
\text { Satisfactorio }\end{array}$ & 0 & $0 \%$ \\
Total & $\mathbf{2 4}$ & $\mathbf{1 0 0 \%}$ \\
\hline
\end{tabular}

La tabla 3 indica que para el $83 \%$ de los emprendedores, la calidad de las actividades de Vinculación con la Sociedad ha sido muy satisfactoria, para el $17 \%$ ha sido satisfactorio; lo cual revela que la Universidad Regional Autónoma de los Andes Quevedo prioriza y atiende las necesidades de la población, a través del suministro de herramientas y formación técnicaadministrativa de calidad, para la generación de emprendimientos de negocios, unidades productivas $\mathrm{y}$ microempresas.

Tabla 4

\section{Expectativas}

\begin{tabular}{lcc}
\hline Descripción & Frecuencia & Porcentaje \\
\hline $\begin{array}{l}\text { Muy } \\
\text { satisfactorio }\end{array}$ & 22 & $92 \%$ \\
\hline Satisfactorio & 2 & $8 \%$ \\
$\begin{array}{l}\text { Poco } \\
\text { satisfactorio }\end{array}$ & 0 & $0 \%$ \\
Nada & 0 & $0 \%$ \\
Satisfactorio & & $\mathbf{1 0 0 \%}$ \\
\hline Total & $\mathbf{2 4}$ & \\
\hline
\end{tabular}

Según los datos presentados en la tabla 4 el $92 \%$ de los encuestados,
UNIANDES_QUEVEDO a través de las actividades de vinculación han cumplido sus expectativas muy satisfactoriamente, mientras que el $8 \%$ indican que ha sido satisfactorio. Dichos resultados muestran que la sociedad tiene plena confianza en los conocimientos que imparte la institución, así como también en los estudiantes y personal docente que forma parte del proyecto de vinculación.

Tabla 5

Aporte del proyecto de vinculación

\begin{tabular}{lcc}
\hline Descripción & Frecuencia & Porcentaje \\
\hline Alto & 24 & $100 \%$ \\
Medio & 0 & $0 \%$ \\
Bajo & 0 & $0 \%$ \\
Total & $\mathbf{2 4}$ & $\mathbf{1 0 0 \%}$ \\
\hline
\end{tabular}

La tabla 5 pone de manifiesto que el $100 \%$ de los beneficiarios declaran que el producto entregado por UNIANDES -QUEVEDO aporta a mejorar su calidad de vida y al desarrollo local. Además, es necesario mencionar que el proyecto de vinculación contribuye al desarrollo personal, económico y social de los beneficiarios, favoreciendo al fortalecimiento del aparato productivo del país.

Tabla 6

Acercamiento de la universidad con su comunidad

\begin{tabular}{lcc}
\hline Descripción & Frecuencia & Porcentaje \\
\hline $\begin{array}{l}\text { Muy } \\
\text { satisfactorio }\end{array}$ & 20 & $83 \%$ \\
\hline Satisfactorio & 4 & $17 \%$ \\
\hline $\begin{array}{l}\text { Poco } \\
\text { satisfactorio }\end{array}$ & 0 & $0 \%$ \\
\hline $\begin{array}{l}\text { Nada } \\
\text { Satisfactorio }\end{array}$ & 0 & $0 \%$ \\
Total & $\mathbf{2 4}$ & $\mathbf{1 0 0 \%}$ \\
\hline
\end{tabular}

En la tabla 6 se expone que el $83 \%$ de los participantes consideran que los 
proyectos de vinculación permiten un acercamiento de la universidad con su comunidad muy satisfactoriamente, mientras que el $17 \%$ indican que es satisfactorio. Es necesario mencionar que en el sector productivo existen numerosos problemas, entre ellos: insuficiente gestión en programas y proyectos basados en políticas públicas nacionales enmarcadas en el Plan Nacional de Desarrollo, que busquen mejorar las capacidades y las potencialidades de la ciudadanía. (salud, educación, capacitación y promoción laboral, emprendimiento productivo)., UNIANDES - QUEVEDO beneficia a grupos vulnerables como los jóvenes, que son actores de la economía popular y solidaria que requieren mayor apoyo por sus condiciones económicas y vida social.

\section{Tabla 7}

\section{Influencia del emprendimiento}

\begin{tabular}{lcc}
\hline Descripción & Frecuencia & Porcentaje \\
\hline Alto & 24 & $100 \%$ \\
Medio & 0 & $0 \%$ \\
Bajo & 0 & $0 \%$ \\
Total & $\mathbf{2 4}$ & $\mathbf{1 0 0 \%}$ \\
\hline
\end{tabular}

Según la tabla 7 el $100 \%$ de encuestados manifiestan que el proyecto de vinculación generación de emprendimientos ejecutado por UNIANDES-QUEVEDO ha influido de manera positiva en su nivel de emprendimiento, esto es posible gracias a la motivación y conocimientos impartidos por parte de estudiantes y docentes participantes.

\section{Tabla 8}

\section{Microempresa en funcionamiento}

\begin{tabular}{lcc}
\hline Descripción & Frecuencia & Porcentaje \\
\hline Si & 7 & $29 \%$ \\
No & 14 & $58 \%$ \\
En proceso & 3 & $13 \%$ \\
Total & $\mathbf{2 4}$ & $\mathbf{1 0 0 \%}$ \\
\hline
\end{tabular}

En la tabla 8 se presenta que el $29 \%$ de beneficiarios han podido ejecutar los emprendimientos en los cantones de Quevedo, Valencia, Buena $\mathrm{Fe} y$ Mocache, estas unidades de negocio corresponden al área comercial y de servicios. El 58\% de emprendedores aún no han podido establecer sus microempresas, ya que para ello esperan que se efectúe un crédito al que han aplicado en BanEcuador. Mientras que al $13 \%$ de los participantes ya les otorgaron el préstamo bancario y en la actualidad se encuentran realizando las actividades pertinentes para aperturar sus unidades productivas.

\section{Tabla 9}

\section{Impacto socioeconómico}

\begin{tabular}{lcc}
\hline Descripción & Frecuencia & Porcentaje \\
\hline $\begin{array}{l}\text { Totalmente } \\
\text { de acuerdo }\end{array}$ & 8 & $33 \%$ \\
$\begin{array}{l}\text { De acuerdo } \\
\text { En }\end{array}$ & 16 & $67 \%$ \\
desacuerdo & 0 & $0 \%$ \\
$\begin{array}{l}\text { Totalmente } \\
\text { en } \\
\text { desacuerdo }\end{array}$ & 0 & $0 \%$ \\
Total & $\mathbf{2 4}$ & $\mathbf{1 0 0 \%}$ \\
\hline
\end{tabular}

La tabla 9 expone que los emprendimientos generados y los que están por establecerse tienen un impacto el desarrollo económico y social de los actores y organizaciones de economía popular y solidaria en los cantones en los que operaran.

\section{DISCUSIÓN}

La investigación realizada permite concluir que las Instituciones de Educación Superior en la actualidad tiene un gran compromiso social, en la cual se involucra a los estudiantes quienes participan y aplican los conocimientos adquiridos en la Universidad en la solución de necesidades y problemas de la comunidad. 
La universidad Regional Autónoma de los Andes UNIANDES- QUEVEDO se encuentra comprometida fielmente con la sociedad, por lo que en aras de vincularse con la misma aporta en forma significativa con sus estudiantes y personal docente, para colaborar en el desarrollo y la productividad empresarial, manteniendo de manera continua la ejecución del proyecto en los diferentes sectores de la colectividad.

A través de la elaboración de planes de negocio se aporta a la búsqueda de nuevas oportunidades de negocio que permita promover el empleo y el crecimiento económico inclusivo $\mathrm{y}$ sostenido de las familias riosenses.

La investigación realizada permite determinar que la implantación de emprendimientos, a través del diseño de un plan de negocio ha contribuido con la productividad y competitividad de los actores y organizaciones de economía popular y solidaria. Se logró la elaboración del 69\% (24) de planes de negocio respecto a la meta trazada (35).

Del total de personas encuestadas, el $29 \%$ han podido ejecutar sus unidades de negocio en el área comercial y de servicios en los cantones: Quevedo, Valencia, Buena Fe y Mocache. El 58\% de emprendedores aún no han podido establecer sus microempresas, ya que para ello esperan que se efectúe un crédito al que han aplicado en una institución financiera estatal.

Así mismo, los beneficiarios manifiestan que el proyecto de vinculación generación de emprendimientos ejecutado por UNIANDES-QUEVEDO ha influido de manera positiva en su nivel de emprendimiento, por lo que consideran este tipo de actividades que ejecuta la universidad tiene un impacto importante en el desarrollo económico y social de los actores y organizaciones de economía popular y solidaria, ya que se atienden las necesidades de la población, a través del suministro de herramientas y formación técnicaadministrativa de calidad, para la generación de emprendimientos de negocios, unidades productivas $\mathrm{y}$ microempresas.

\section{REFERENCIAS BIBLIOGRÁFICAS}

Blanco , A., Mercado, C., \& Prado, A. (2012). Perfil y motivación de la juventud emprendedora española. Estudios de Juventud, 23.

Calzada, B. (2012). El emprendimiento en España. Una perspectiva histórica. Estudios de Juventud, 15.

De Aparicio , X., Chininin , M., \& Toledo, O. d. (2017). El Rol de la Vinculalción en la Integración de las Funciones Sustantivas de la Universidad Metropolitana del Ecuador. Revista Científica de la Universidad de Cienfuegos, 39.

Delfín , F. L., \& Acosta , M. P. (2016). Importancia y análisis del desarrollo empresarial . Pensamiento y Gestión, 187.

Gaete, R. (2011). Responsabilidad Social Universitaria: Una Nueva Mirada a la Relación de la Universidad con la Sociedad desde la Perspectiva de las Partes Interesadas. Un Estudio de Caso. España. Obtenido de https://uvadoc.uva.es/bitstream/1 0324/923/1/TESIS148120417.pdf

Jaramillo , L. (2008). Emprendimiento: Concepto básico en competencias . Instituto de Estudios en Educación . 
Maass, M., \& Sabulsky, G. (2015). La vinculación como estrategia de formación en la educación superior. Áreas de Humanidades y Ciencias Sociales y de Artes, Arquitectura y Diseño. Red Innova Cesal , 89.

\section{SECRETARÍA NACIONAL DE}

PLANIFICACIÓN Y

DESARROLLO-SENPLADES. (2017). Secretaría Nacional de Planificación y Desarrollo.

Obtenido de

http://www.planificacion.gob.ec/

wp-

content/uploads/downloads/2017

/10/PNBV-26-OCT-

FINAL_0K.compressed1.pdf 